\title{
Dissolution Behavior of Gold in Alkaline Media Using Thiourea
}

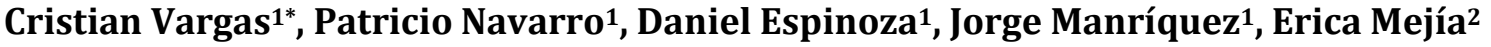 \\ ${ }^{1}$ Departamento de Ingeniería Metalúrgica, Universidad de Santiago de Chile, Avda, Libertador Bernardo O'Higgins, Santiago, \\ Chile \\ ${ }^{2}$ Grupo de Investigación GIIAM, Facultad de Ingeniería, Institución Universitaria Pascual Bravo, Medellín, Colombia \\ Email: *cristian.vargas@usach.cl
}

How to cite this paper: Vargas, C., Navarro, P., Espinoza, D., Manríquez, J. and Mejía, E. (2019) Dissolution Behavior of Gold in Alkaline Media Using Thiourea. International Journal of Nonferrous $\mathrm{Me}$ tallurgy, 8, 1-8.

https://doi.org/10.4236/ijnm.2019.81001

Received: December 10, 2018

Accepted: December 26, 2018

Published: December 29, 2018

Copyright (C) 2019 by authors and Scientific Research Publishing Inc. This work is licensed under the Creative Commons Attribution International License (CC BY 4.0).

http://creativecommons.org/licenses/by/4.0/

\section{Open Access}

\begin{abstract}
In this work the dissolutive behavior of gold in alkaline medium using thiourea (TU), under different variables, was studied in a theoretical and experimental way, in order to determine the conditions under which it is feasible to dissolve gold in thiourea-alkaline medium. A thermodynamic study was conducted by chemical speciation using the method of Rojas-Hernández, together with an electrochemical study where the electric potential was swept in the anodic direction. The main results of the thermodynamic study were that formamidine disulfide (FDS) and sulfinic compounds (S.C.) prevail at alkaline $\mathrm{pH}$; by increasing the initial concentration of thiourea at alkaline $\mathrm{pH}$, the presence of the gold complex is almost zero for any initial concentration of thiourea. By including sodium sulfite in the gold-thiourea system, it was possible to obtain the $\mathrm{Au}(\mathrm{I})$ - TU complex at alkaline $\mathrm{pH}$, with a presence of $95.13 \%$. Electrochemical tests allowed verifying that in the absence of sodium sulfite the dissolution of gold in an alkaline medium is very slow but adding sodium sulfite improvements become evident in the dissolution of the metal. Therefore, sodium sulfite catalyzes the gold dissolution process and stabilizes the thiourea. With this study it was possible to establish the feasibility of using thiourea in an alkaline medium for the dissolution of gold, and the conditions under which it is possible to dissolve the gold in that medium. With these fundamentals and conditions, it is now possible to move forward to test this system for minerals and/or concentrates containing gold.
\end{abstract}

\section{Keywords}

Gold, Alkaline, Thiourea, Dissolution Behavior, Sodium Sulfite

\section{Introduction}

The use of thiourea $\left(\mathrm{SC}\left(\mathrm{NH}_{2}\right)_{2}\right)$ (TU) has been studied extensively, mainly be- 
cause it is not harmful to the environment, nontoxic to human beings, and the dissolution of gold in thiourea solutions is much faster than in cyanide solutions. The dissolution of gold in thiourea solutions was reported for the first time in 1941, and it was continued in 1960 [1]. It has been reported that gold dissolves more efficiently with thiourea in acid than in alkaline solution. Thiourea is unstable above $\mathrm{pH}=4.3$, and its degradation is also caused by oxidation. Thiourea is oxidized to formamidine disulfide (FDS), which is an active oxidant for dissolving gold. FDS is also oxidized to various intermediary species whose final product is elemental sulfur, which causes the passivation of precious metals, and their dissolution tends to stop [2] [3].

In the leaching of gold with acid TU solutions there are several problems, such as a greater consumption of $\mathrm{TU}$ during the redissolution of $\mathrm{Au}$, equipment corrosion in acid solution, low selectivity of the Au leaching reaction, and complicated reagent regeneration and purification procedures. To overcome the above inconveniences, the leaching of $\mathrm{Au}$ with an alkaline TU solution has been proposed, and some research work has been carried out [4] [5]. Since the leaching of $\mathrm{Au}$ with alkaline TU solution is still in the research and development stage, it is necessary to make an adequate study of the thermodynamic aspects of the $\mathrm{Au}-\mathrm{TU}$ system under different $\mathrm{pH}$ and ligand concentration conditions that can clarify and establish those required for the operation's success. Therefore, this paper involved chemical and electrochemical studies meant to achieve a better understanding of the gold-thiourea system in an alkaline environment, and to establish the most favorable conditions for the leaching.

\section{Methodology}

\subsection{Chemical Speciation}

The chemical speciation diagrams are constructed to show the relation of the different chemical species (thiourea and gold-thiourea) either as a function of $\mathrm{pH}$ or of ligand concentration and establishing their predominant behavior in solution. The construction of those diagrams in this study is based on the method of Rojas-Hernández [6] [7], which considers the formation of complexes in which a metal ion $\mathrm{M}$ reacts with ligand $\mathrm{L}$ to form the complex ML. For these reactions the formation equilibrium or stability constants are considered. The stability constants used in the construction of the diagrams are shown in Table 1.

\subsection{Electrochemical Tests}

Linear potential scans were made, from the rest potential to $1000 \mathrm{mV}$ in the anodic direction, at a scanning rate of $10 \mathrm{mV} / \mathrm{s}$. To determine the electrochemical behavior of the gold, tests were run under different $\mathrm{pH}$, thiourea concentration, and additive presence and content.

The solutions used were prepared with distilled and deionized water, with analytical grade reagents. 
Table 1. Stability constants of the studied system.

\begin{tabular}{cccc}
\hline Metal & Ligand & Equilibrium reaction & Log K \\
\hline \multirow{2}{*}{$\mathrm{Au}(\mathrm{I})$} & $\mathrm{TU}\left(\mathrm{SC}\left(\mathrm{NH}_{2}\right)_{2}\right.$ & $2 \mathrm{SC}\left(\mathrm{NH}_{2}\right)_{2}=\left[\mathrm{SC}(\mathrm{NH}) \mathrm{NH}_{2}\right]_{2}+2 \mathrm{H}^{+}+2 \mathrm{e}$ & -4.3 \\
& $\mathrm{TU}\left(\mathrm{SC}\left(\mathrm{NH}_{2}\right)_{2}\right.$ & $\mathrm{Au}^{+}+2 \mathrm{SC}\left(\mathrm{NH}_{2}\right)_{2}=\mathrm{Au}\left[\mathrm{SC}\left(\mathrm{NH}_{2}\right)_{2}\right]_{2}^{+}$ & 23.3 \\
\hline
\end{tabular}

All the electrochemical tests were performed with a conventional three-electrode system consisting of a gold working electrode, a platinum wire as counter electrode, and a $\mathrm{Ag} / \mathrm{AgCl}$ reference electrode.

The electrochemical measurements were made with a model PGP-201 Radiometer Potentiosat/Galvanostat controlled by the Voltamaster 4.0 software. All the potential values are referred to the standard hydrogen electrode (SHE).

\section{Results and Discussion}

\subsection{Thiourea Speciation}

Experimental evidence has been found that TU is degraded irreversibly to formamidine disulfide (FDS) and sulfinic compounds (S.C.), reaching elemental sulfur as final product. To confirm the above and determine its stability under different conditions, the speciation diagrams for this ligand were constructed. Figure 1(a) and Figure 1(b) present the speciation diagrams of TU as a function of the concentration of thiourea at alkaline $\mathrm{pH}$.

It is seen that at $\mathrm{pH}$ above 10 there is no more TU in the system, DSF starts degrading, and sulfinic compounds become predominant at $\mathrm{pH}$ greater than 12.5. In Figure $1(\mathrm{~b})$ it is also seen that at alkaline $\mathrm{pH}$ the DSF predominates up to $\mathrm{p}[\mathrm{TU}]=2.3$. As the initial TU concentration is reduced, the presence of DSF decreases, causing an increase of the presence the sulfinic compounds. Furthermore, it can also be confirmed that at alkaline $\mathrm{pH}$ there is no presence of $\mathrm{TU}$ in the system.

\subsection{Au(I)-TU System Speciation}

Gold can form only one complex with thiourea, namely $\mathrm{Au}\left[\mathrm{SC}\left(\mathrm{NH}_{2}\right)_{2}\right]_{2}^{+}$. The behavior of the Au-TU system as a function of $\mathrm{pH}$ and of the TU concentration is shown in Figure 2(a) and Figure 2(b). The Au-TU complex predominates up to $\mathrm{pH}$ 8.8; from that value up to about 12.4, DSF predominates. Above $\mathrm{pH} 12.4$ the presence of DSF decreases, sulfinic compounds become predominant, and the presence of the Au-TU complex is not detected in the system.

Figure 2(b) shows that at alkaline $\mathrm{pH}$ the presence of $\mathrm{Au}(\mathrm{I})$ and of the $\mathrm{Au}-\mathrm{TU}$ complex is almost imperceptible over the whole range of $\mathrm{p}$ [TU], with DSF as the predominant compound from high thiourea concentrations $(\mathrm{p}[\mathrm{TU}]=-1.0)$ to $\mathrm{p}[\mathrm{TU}]=3.3$, at which point the change in predominance occurs, with sulfinic compounds starting to become preponderant at the expense of DSF.

The behavior of this system in the presence of sodium sulfite was also studied. This compound stabilizes TU over the whole $\mathrm{pH}$ range, so it is very important in the stability of the $\mathrm{Au}-\mathrm{TU}$ complex. Figure 3 shows the speciation diagrams of the Au-TU-sodium sulfite system. 


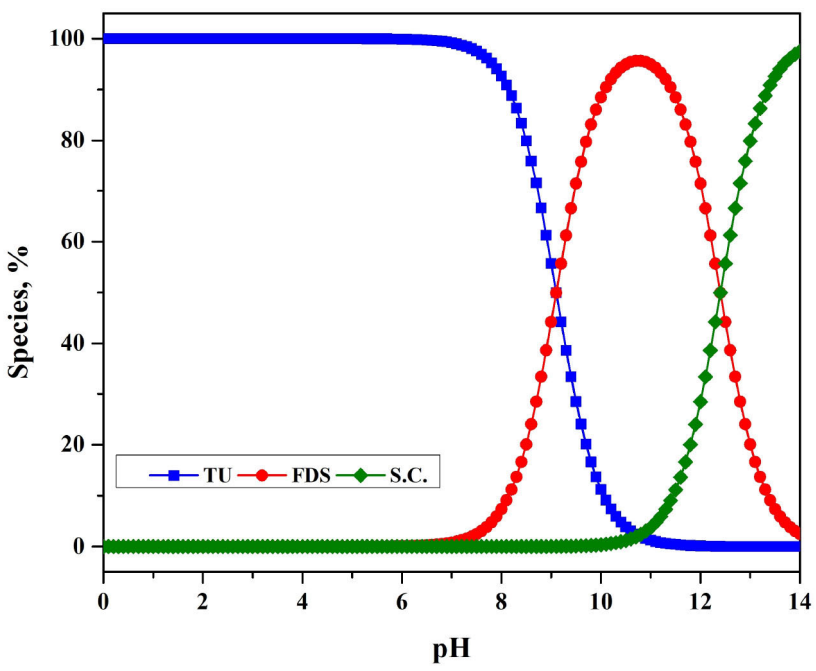

(a)

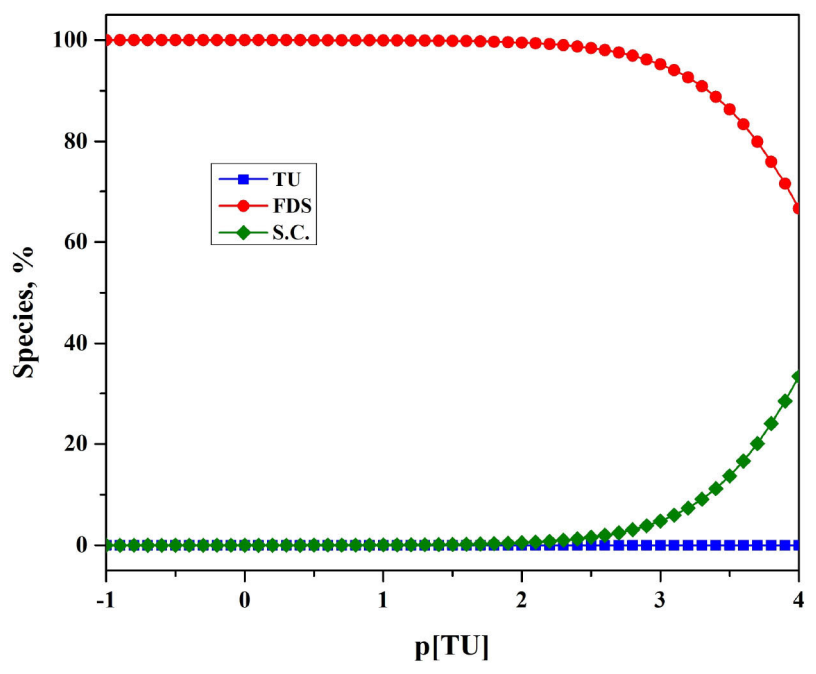

(b)

Figure 1. Speciation of thiourea at 25 as a function of $\mathrm{pH}(\mathrm{a})$ and of thiourea concentration (b).

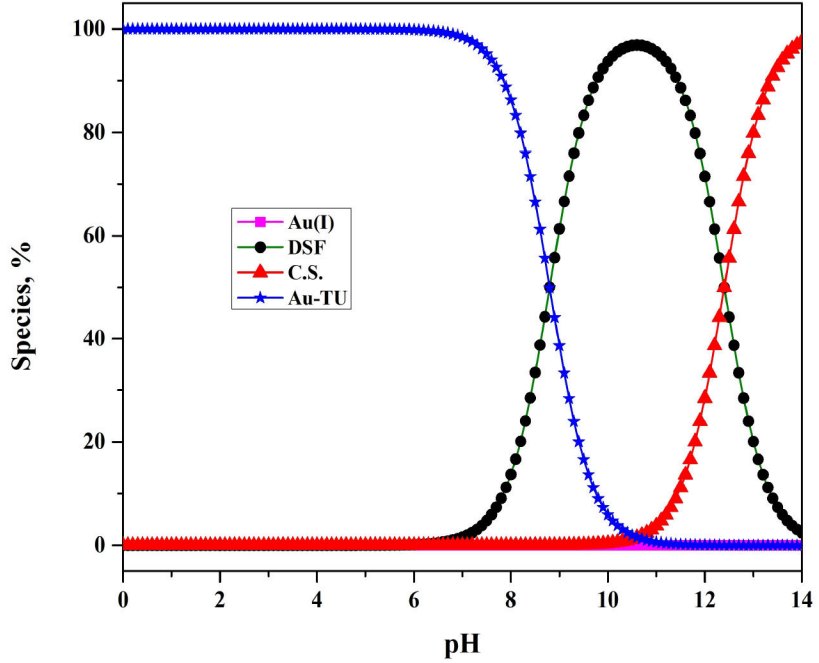

(a)

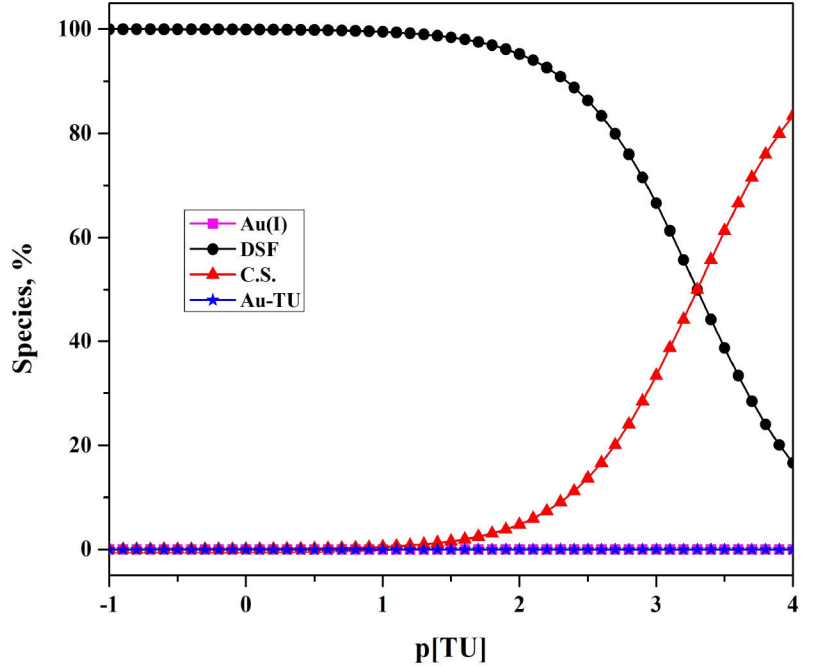

(b)

Figure 2. Speciation of the Au-TU system at $25^{\circ} \mathrm{C}$ as a function of $\mathrm{pH}$ (a) and of TU concentration (b).

It is seen that the Au-TU complex predominates up to $\mathrm{pH}$ 13.7. At higher $\mathrm{pH}$ TU starts predominating; its presence is due to the stabilizing action of sulfite, preventing its irreversible oxidation. Figure $3(\mathrm{~b})$ shows that as TU concentration increases, the predominant species is always the Au-TU complex.

\subsection{Electrochemical Behavior of the Au-TU System}

Figure 4(a) and Figure 4(b) present the electrochemical behavior of $\mathrm{Au}$ as a function of $\mathrm{pH}\left(0.1 \mathrm{M}\right.$ thiourea and $\left.25^{\circ} \mathrm{C}\right)$ and of $\mathrm{TU}$ concentration $(\mathrm{pH}=12.5$ and $25^{\circ} \mathrm{C}$ ).

It is seen that in the Au-TU system, at acid $\mathrm{pH}(1.4)$ two peaks or singularities appear, pointing to the occurrence of some transformation in the system. The 


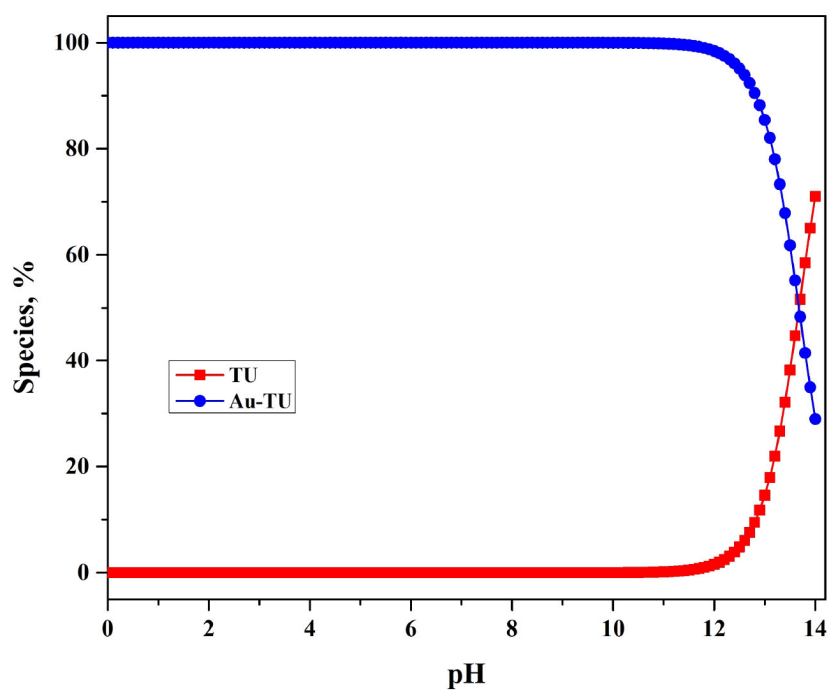

(a)

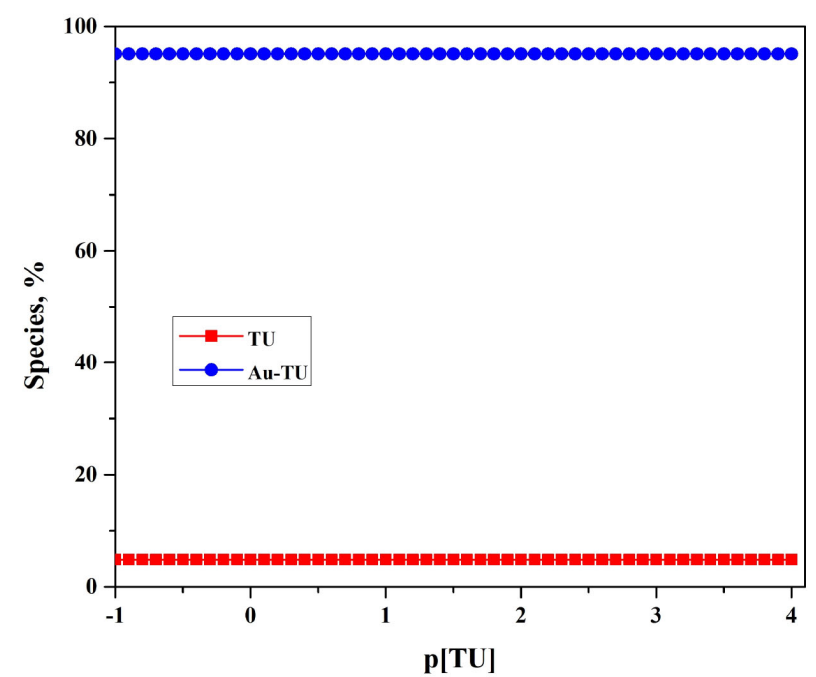

(b)

Figure 3. Speciation of the Au-TU-sodium sulfite system at $25^{\circ} \mathrm{C}$ as a function of $\mathrm{pH}$ (a) and of TU concentration (b).

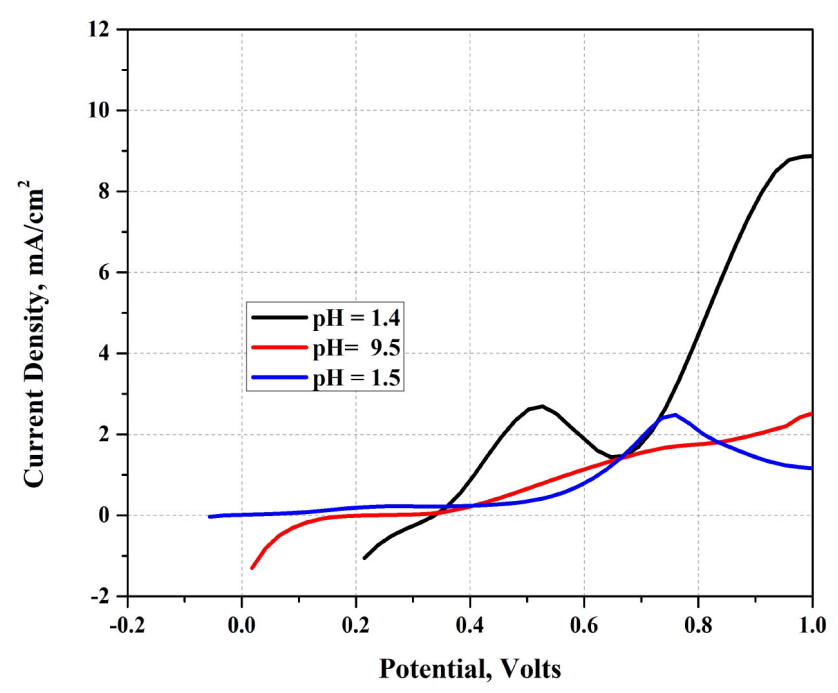

(a)

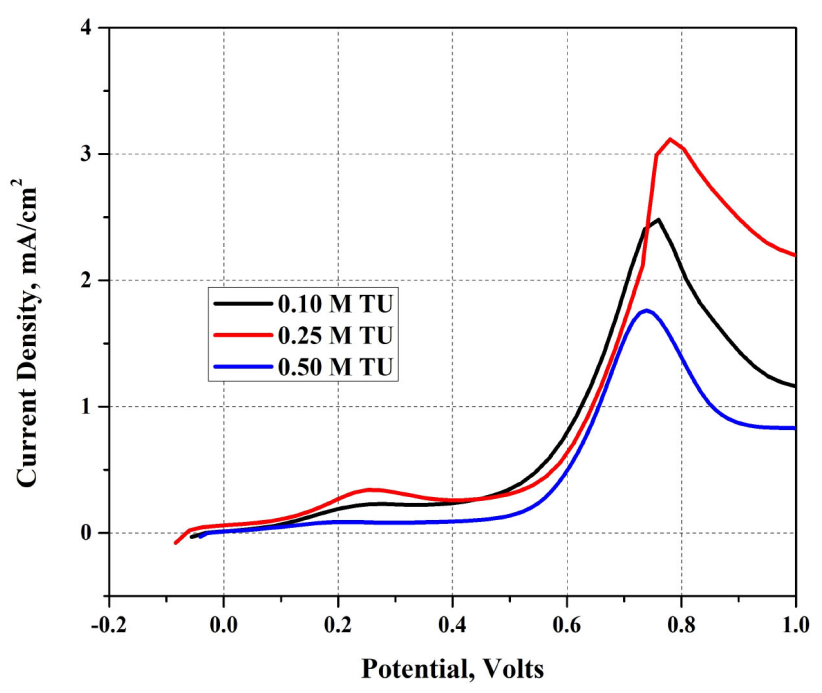

(b)

Figure 4. Linear polarization curves for a gold electrode as a function of $\mathrm{pH}$ (a) and of TU concentration (b).

first peak $(0.52 \mathrm{~V})$ would correspond to the formation of the Au-TU complex, while the second $(0.95 \mathrm{~V})$ would indicate the transformation of TU into DSF. These results are like those obtained by Bolzán et al. [8] and Yang et al. [9], who proposed the occurrence of the following reactions.

$$
\begin{gathered}
\mathrm{Au}+2 \mathrm{SC}\left(\mathrm{NH}_{2}\right)_{2} \Leftrightarrow \mathrm{Au}\left[\mathrm{SC}\left(\mathrm{NH}_{2}\right)_{2}\right]_{2}^{+}+\mathrm{e}, \mathrm{E}^{\circ}=0.38 \mathrm{~V}_{\mathrm{NHE}} \\
2 \mathrm{SC}\left(\mathrm{NH}_{2}\right)_{2} \Leftrightarrow \mathrm{NH}_{2}(\mathrm{NH}) \mathrm{CSSC}(\mathrm{NH}) \mathrm{NH}_{2}+2 \mathrm{H}^{+}+2 \mathrm{e}, \mathrm{E}^{\circ}=0.42 \mathrm{~V}_{\mathrm{NHE}}
\end{gathered}
$$

At $\mathrm{pH} 9.5$ no singularity is seen, but not so at $\mathrm{pH} 12.5$, when the peak of the DSF formation reaction $(0.76 \mathrm{~V})$ appears. Furthermore, a greater electrochemical activity under acid conditions $(\mathrm{pH}=1.4)$ is seen than at more alkaline $\mathrm{pH}$ 


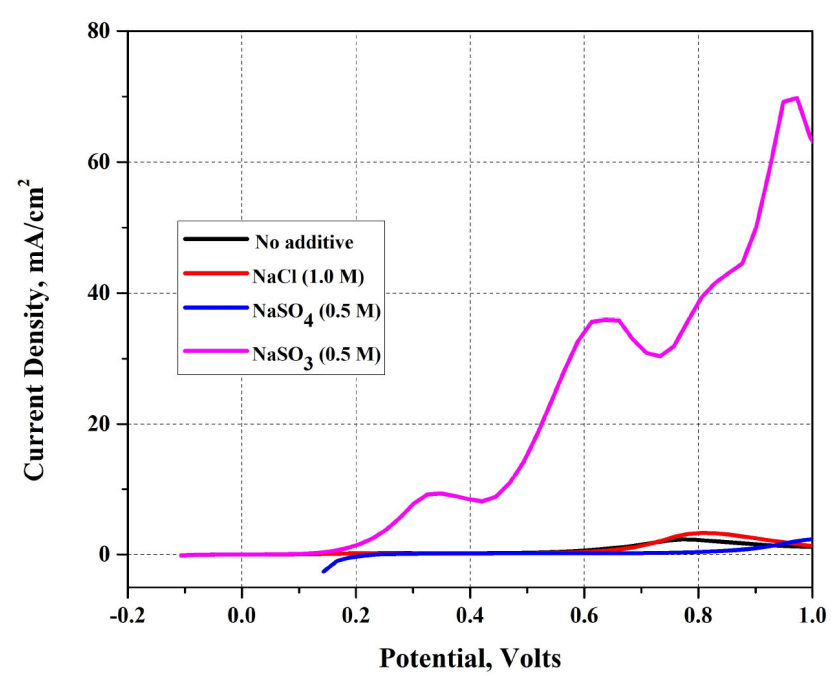

(a)

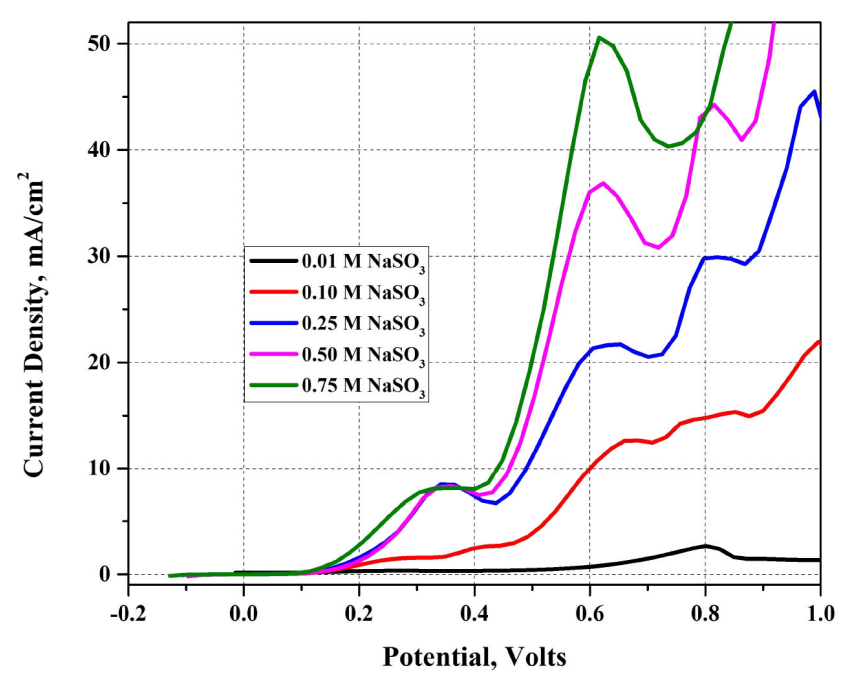

(b)

Figure 5. Linear polarization curves for a gold electrode in the presence of different additives (a), and of the concentration of sodium sulfite (b).

(9.5 and 12.5), and at $\mathrm{pH} 12.5$ it is not possible to see the typical singularity that indicates the formation of the Au-TU complex, confirming what had been shown previously for chemical speciation. Comparing the curves shown in Figure 4(b), it is seen that for all the TU concentrations that were studied, the singularities occur at similar potentials, so the variation of the TU concentration at $\mathrm{pH} 12.5$ has no influence on the potential at which the system's chemical transformations occur.

The effect of the presence of additives on the electrochemical behavior of gold in an alkaline medium with thiourea was also determined. Figure 5(a) shows that the presence of sodium sulfite increases visibly the electrochemical activity of gold compared to the other solutions that were studied. Therefore, sodium sulfite allows obtaining the Au-TU complex defining an important condition in the use of thiourea for leaching gold in an alkaline medium. Figure 5(b) shows that the dissolution of gold increases as the presence of sodium sulfite also increases.

\section{Conclusions}

The speciation studies made allowed establishing that regardless of the TU concentration and in an alkaline medium, the TU is not predominant in the system because it decomposes irreversibly until it forms sulfinic compounds. This means that there is no thiourea available for complexing the gold, and that is why under alkaline conditions and for any TU concentration it is not possible to obtain the Au-TU complex as the predominant species. However, the addition of sodium sulfite stabilizes the thiourea, allowing the predominance of the Au-TU complex in an alkaline medium.

The electrochemical studies showed different current peaks at given potential 
values, which represent the transformations undergone by the species present in the system. In an alkaline medium there was no evidence of the formation of the Au-TU complex. The concentration of thiourea does not affect the potential at which the singularities occur, however, a greater TU concentration does not imply a higher rate of dissolution. The addition of sodium sulfite to the thiourea solution showed clearly the formation peak of the $\mathrm{Au}-\mathrm{TU}$ complex, greatly increasing the anodic current of the gold electrode, speeding up its dissolution.

The results obtained allow establishing certain conditions of use of thiourea in alkaline medium to dissolve gold. The application of this system for minerals and/or concentrates required additional study regarding the effect and interaction of some impurities, as well as the determination of kinetic mechanisms and parameters of interest for a better understanding and optimization of the process.

\section{Acknowledgements}

Support by the Dirección de Investigaciones Científicas y Tecnológicas of the Universidad de Santiago de Chile (DICYT) through project 051314VR is gratefully acknowledged.

\section{Conflicts of Interest}

The authors declare no conflicts of interest regarding the publication of this paper.

\section{References}

[1] Lin, J. and Huarng, J. (1994) Electrochemical Stripping of Gold from Au-Ni-Cu Electronic Connector Scrap in an Aqueous Solution of Thiourea. Journal of the Applied Electrochemistry, 24, 157-165. https://doi.org/10.1007/BF00247788

[2] Groenewald, T. (1976) The Dissolution of Gold in Acidic Solutions of Thiourea. Hydrometallurgy, 1, 277-290. https://doi.org/10.1016/0304-386X(76)90004-9

[3] Schulze, R. (1984) New Aspects in Thiourea Leaching of Precious Metals. JOM, 36, 62-65. https://doi.org/10.1007/BF03338478

[4] Zhang, C., Chai, L., Zhong, H., Masazumi, O. and Ryoichi, I. (1997) Selective Dissolution of Gold in an Alkaline Thiourea Solution by Electrolysis. Journal of Central South University of Technology, 4, 73-78. https://doi.org/10.1007/s11771-997-0001-8

[5] Dawei, W., Liyuan, C., Ryoichi, I. and Masazumi, O. (1999) Gold Leaching in an Alkaline Thiourea Solution. Journal of the Electrochemical Society, 146, 559-563. https://doi.org/10.1149/1.1391643

[6] Rojas-Hernández, A., Ramírez, M., Ibáñez, J. and González, I. (1991) Construction of Multicomponent Pourbaix Diagrams Using Generalized Species. Journal of the Electrochemical Society, 138, 365-371. https://doi.org/10.1149/1.2085590

[7] Rojas-Hernández, A., Ramírez, M., González, I. and Ibáñez, J. (1995) Predominance-Zone Diagrams in Solution Chemistry: Dismutation Processes in Two-Component Systems (M-L). Journal of Chemical Education, 72, 1099-1105. https://doi.org/10.1021/ed072p1099

[8] Bolzán, A., Piatti, R. and Arvia, A. (2003) Electrochemical Processes at Gold Thiou- 
rea Containing Aqueous Acid Solution Interfaces. Journal of Electroanalytical Chemistry, 552, 19-34. https://doi.org/10.1016/S0022-0728(03)00020-2

[9] Yang, X., Moats, M. and Miller, J.D. (2010) The Interaction of Thiourea and Formamidine Disulfide in the Dissolution of Gold in Sulfuric Acid Solutions. Minerals Engineering, 23, 698-704. https://doi.org/10.1016/j.mineng.2010.04.006 